\title{
Femoral Placement of Totally Implantable Venous Access Ports in Patients with Bilateral Breast Cancer
}

\author{
Transfemorale Portimplantation beim beidseitigen Mammakarzinom
}

Authors

Affiliations
V. Almasi-Sperling ${ }^{1}$, S. Hieber ${ }^{1}$, J. Lermann ${ }^{2}$, O. Strahl ${ }^{2}$, M. W. Beckmann ${ }^{2}$, W. Lang ${ }^{1}$, T. A. Sagban ${ }^{1}$

${ }^{1}$ Department of Vascular Surgery, Friedrich Alexander University Erlangen, Erlangen

2 Department of Obstetrics and Gynecology, Friedrich Alexander University Erlangen, Erlangen
Key words

- breast cancer

- femoral vein

- thrombosis

venous access port

Schlüsselwörter

- Brustkrebs

- Femoralvene

- Thrombose

๑ venöses Portsystem $\begin{array}{ll}\text { received } & 15.6 .2015 \\ \text { revised } & 5.8 .2015 \\ \text { accepted } & 21.8 .2015\end{array}$

Bibliography

DOI http://dx.doi.org/

10.1055/s-0035-1558173

Geburtsh Frauenheilk 2016; 76:

53-58 ๑ Georg Thieme

Verlag KG Stuttgart · New York . ISSN 0016-5751

\section{Correspondence}

\section{Dr. Tolga Atilla Sagban, MD}

Friedrich Alexander University Erlangen

Department of Vascular Surgery

Krankenhausstraße 12

91054 Erlangen

drsagban@yahoo.de

\section{Abstract}

$\nabla$

Purpose: Aim of this study was to determine the rate of complications following femoral placement of totally implantable venous access ports (f-TIVAP) in women with bilateral breast cancer, with a special focus on long-term function, deep vein thrombosis (DVT), and port infection.

Methods: 73 patients with bilateral breast cancer treated between October 2000 and January 2013 with placement of an f-TIVAP using a transfemoral approach were retrospectively reviewed. All patients were followed up, and all complications of f-TIVAP were recorded.

Results: The median age was 62.5 years (range: 35-86 years). Four patients received f-TIVAP under local anesthesia, and 69 underwent placement under general anesthesia. Mean follow-up was 33.7 months (SD 25.9; range: 0.293.5 months). Complications over the entire period of observation included infections in $21 \%$, DVT in $19 \%$ and catheter occlusion in $12 \%$. Patients receiving chemotherapy who developed leukopenia were more likely to experience DVT at the access site $(p=0.037)$. There was a trend towards a higher infection rate when the device was used more often ( $\mathrm{p}=0.084)$.

Conclusion: Although the rates of complications in the longer term, especially device infections and DVTs, appeared to be relatively high, TIVAP implantation using femoral vein access is recommended in patients with bilateral breast cancer not suitable for cephalic vein cut-down.

\section{Zusammenfassung \\ $\nabla$}

Einleitung: Ziel dieser Studie war es, die Komplikationsraten von transfemoralen Portsystemen in die Femoralvene (f-TIVAP) bei Patienten mit beidseitigem Mammakarzinom hinsichtlich der Langzeitfunktion, Ausbildung einer tiefen Venenthrombose (TVT) und Portinfektion zu beurteilen. Material und Methodik: 73 Patientinnen mit beidseitigem Mammakarzinom wurden zwischen Oktober 2000 und Januar 2013 mit einem f-TIVAP über einen transfemoralen Zugang zur Therapie versorgt und retrospektiv beurteilt. Alle Patientinnen wurden nachuntersucht und Komplikationen der f-TIVAP wurden aufgezeichnet.

Ergebnisse: Das Durchschnittsalter betrug 62,5 Jahre (min. 35, max. 86 Jahre). Vier Patientinnen erhielten eine f-TIVAP in Lokalanästhesie, 69 in Allgemeinanästhesie. Die mittlere Nachuntersuchungszeit lag bei 33,7 Monaten (SD 25,9; min. 0,2, max. 93,5 Monate). Komplikationen über die gesamte Nachuntersuchungszeit waren: Portinfekte in $21 \%$, in $19 \%$ TVT und in $12 \%$ Portkatheterverschluss. Patientinnen unter Chemotherapie, die eine Leukopenie entwickelten, waren öfter durch eine TVT an der Portkatheter-Implantationsseite betroffen $(p=0,037)$. Ein Trend für eine höhere Infektrate des Portsystems in der Leiste konnte bei öfterem Gebrauch ermittelt werden $(\mathrm{p}=0,084)$.

Diskussion und Fazit: Obwohl Langzeitkomplikationen wie Portinfektionen und TVT relativ häufig aufzutreten scheinen, ist die TIVAP-Implantation über die Femoralvene durch einen Leistenzugang empfehlenswert bei Patienten mit beidseitigem Mammakarzinom, die für eine Portimplantation über die V. cephalica nicht infrage kommen. 


\section{Introduction}

$\nabla$

Totally implantable venous access ports (TIVAP) are crucial for long-term parenteral therapy, e.g. for chemotherapy (CTX), parenteral nutrition and many other treatments $[1,2]$. These ports provide permanent access to the central venous system for longer endovenous treatments [3]. The standard access for both the percutaneous and the surgical approach is via the subclavian or cephalic vein. The tip of the catheter is placed either into the superior vena cava or the right atrium. The chamber is located in the subcutaneous tissue of the anterior chest wall [1]. Bilateral obstruction of the subclavian or brachiocephalic vein is a major reason for attempting to obtain long-term access to the inferior vena cava from a distal vein $[4,5]$. However, the cephalic vein cutdown approach can be difficult in patients with small cephalic veins or venous thrombosis. The cephalic vein cut-down approach is a difficult technique to perform in patients with a history of synchronous bilateral breast surgery, who may have suffered intraoperative injury to the cephalic veins or have postoperative adhesions potentially causing venous thrombosis or making it difficult to locate the cephalic veins. The cut-down method can also increase the possibility of arm swelling in these patients. Many patients with bilateral breast cancer treated with surgery and radiotherapy are sensitive to manipulations of the chest wall, experiencing discomfort when a venous port system is located close to the pretreated breast. In selected patients with bilateral breast cancer, a TIVAP placed via a femoral vein access (f-TIVAP) may be preferable to further trauma after breast surgery or radiation therapy [6]. However, the risk of deep vein thrombosis (DVT) and device infection could be higher due to the location of the access site. This study reports the results of f-TIVAP as a preferred means of venous access in patients with bilateral breast cancer with a special focus on long-term function of the port, DVT and port infection.

\section{Methods}

$\nabla$

\section{Patient recruitment}

73 patients with bilateral breast cancer treated between October 2000 and January 2013 with placement of an f-TIVAP using a transfemoral approach were retrospectively reviewed. The data analysis was approved by the local ethics committee, and informed consent was obtained from each patient. Indications for f-TIVAP implantation were CTX for bilateral breast cancer and, in selected cases, the additional need for parenteral nutrition or continuous intravenous infusion for pain relief. The therapy of all patients was based on current guidelines after presentation of the case to an institutional interdisciplinary board.

\section{Surgical procedure}

Access surgery was standardized with exposure of the greater saphenous or common femoral vein in the groin under general anesthesia ( Fig. 1 a). The catheter tip was positioned in the inferior vena cava with $\mathrm{C}$-arm fluoroscopic control at the level located between the 3 rd and 4 th lumbar vertebra ( 0 Fig. 1 b). The chamber of the device was placed in a subcutaneous pocket at the proximal anterior thigh. It was filled routinely after skin closure with $2 \mathrm{~mL}$ of a solution of $0.9 \%$ heparinized saline ( Figs. $1 \mathrm{c}$ and d). Intravenous single-shot antibiotic prophylaxis was administered before implantation.

\section{Follow-up}

All patients were treated in the Department of Obstetrics and Gynecology, making the data on punctures and irrigations prospectively available from patients' records. DVT of the access vein was diagnosed by determination of D-dimer in patients' blood samples, duplex ultrasound, computed tomography/magnetic resonance tomography, and by venography in some cases; however there was no routine examination of all patients during follow-up. Infection of the access device was suspected based on typical clinical signs (increased pain, redness, swelling, warmth around the port pocket, secretion of pus at the puncture site, visible port chamber). Microbiological testing of blood samples obtained either from the port catheter or from peripheral blood was done to prove f-TIVAP infection. The date of last follow-up in the clinic, death of the patient, and removal of the device were defined as endpoints of follow-up. There was no standardized routine procedure for the scheduled removal of the port after treatment.

\section{Statistical analysis}

Continuous variables were summarized as the mean and standard deviation if normally distributed. Categorical variables were presented as numbers and percentages, and continuous variables were analyzed parametrically using the t-test.

A univariate assessment of categorical variables was performed using the Chi-square test and Fisher's exact test, as needed. A value of $p<0.05$ was considered statistically significant. The data were analyzed using SPSS for Windows, version 20.0 (SPSS, Inc., Chicago, IL, USA).

\section{Results \\ $\nabla$}

\section{Description of study population}

A total of 73 female patients with bilateral breast cancer received an f-TIVAP in our hospital. The median age was 62.5 years (SD 11.4, range: 35-86 years). Four patients received f-TIVAP under local anesthesia, and 69 patients had port placement under general anesthesia. $13 / 73$ patients (18\%) had previously had a TIVAP, two patients of whom had had two previous TIVAPs. These earlier TIVAPs were placed in the right cephalic vein in four patients and in the left cephalic vein in a further four patients. In each case, the device was implanted in the left or right subclavian vein; in one woman it was implanted in the left femoral vein. Two patients, who had had two prior TIVAPs, had placement via the left cephalic vein and left subclavian vein or right femoral vein. $\bigcirc \mathrm{Ta}-$ ble 1 shows the number and location of these earlier f-TIVAPs together with the demographic data.

\section{Surgical procedures and complications}

Mean time of surgery was 24.5 minutes (SD 12.6 minutes, range: $6-75)$. Surgery site was the right groin in 63 patients (86\%) and the left side in ten women (14\%). In 68 of 73 cases, access was obtained directly via the main trunk of the greater saphenous vein or one of its side branches (93\%). In five patients the catheter was inserted via a venotomy of the common femoral vein (7\%). Revision was required in one case ( $1 \%$ ) because of a hematoma in the port pocket. Another patient ( $1 \%$ ) required revision surgery after 28 months because of leakage of one device. There was no mortality caused by f-TIVAP implantation. 


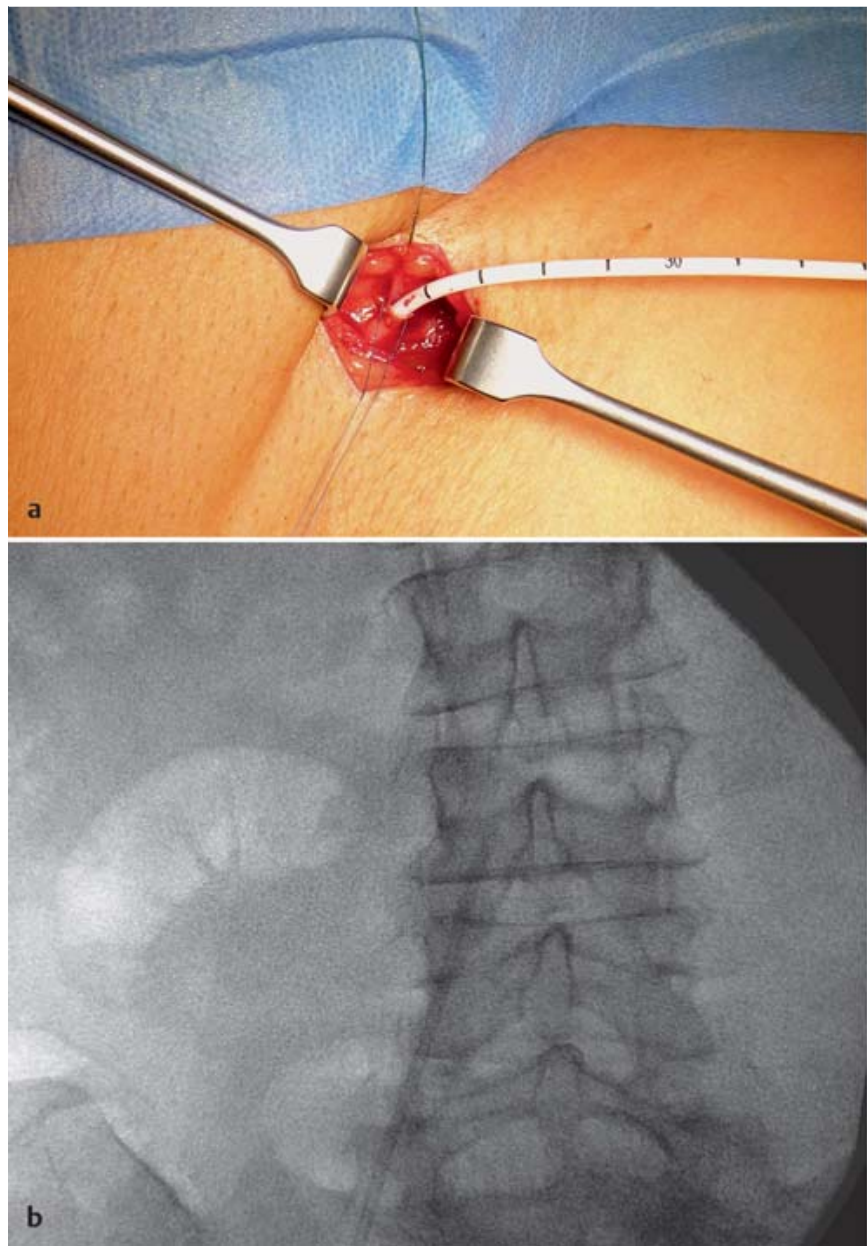

Fig. $1 \mathbf{a}$ to $\mathbf{d}$ Exposure of the greater saphenous or common femoral vein in the groin (a); positioning of the catheter tip in the inferior vena cava under $\mathrm{C}$-arm fluoroscopic control at the level located between the 3rd and 4th lum-

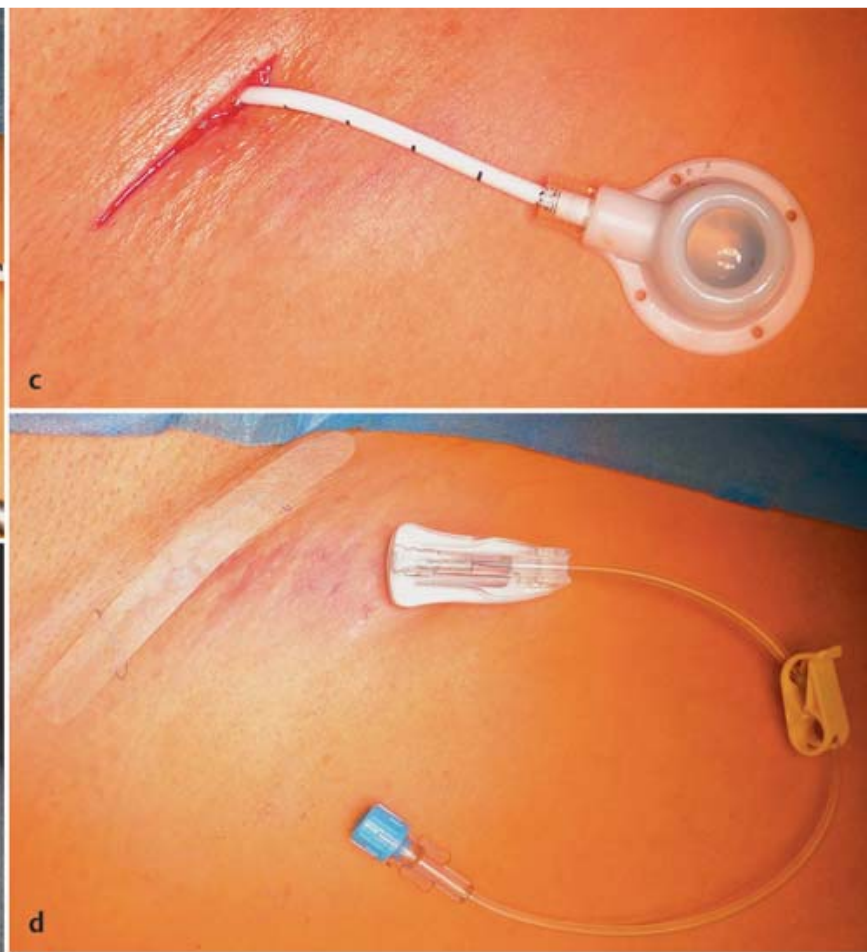

bar vertebra (b); placement of the port chamber in a subcutaneous pocket at the proximal anterior thigh (c and $\mathbf{d}$ ).
Long-term function of f-TIVAP after implantation

Mean follow-up was 33.7 months (SD 25.9 months; range 0.293.5). The average time of f-TIVAP left in situ was 647 days (SD 595 days; range: $6-2495)$. In $38 \%$ of patients (28/73) the f-TIVAP was removed because treatment had ended, while in 45/73 (62\%) the f-TIVAP was left in situ. In all cases except one, CTX was administered through the f-TIVAP. The mean number of punctures of the port system for CTX per patient was 10.3 (SD 6.0; range: 1-30 punctures); flushing with heparinized saline was performed on average 24.9 times per patient (SD 15.2; range 2-75). Nine catheters became occluded during the period of observation (12\%).

\section{Infection as a complication of f-TIVAP caused by puncture}

A total of $15 / 73$ patients (21\%) suffered an infection of the device; in eight women, infection was determined based on clinical signs $(11 \%)$. However, bacteria were verified only in seven of 15 cases (47\%). The most common types of bacteria were skin flora-derived types such as Staphylococcus aureus (in 3 patients), Staphylococcus epidermidis (in 3 patients) and Streptococcus pyogenes (in 1 patient). On average, infectious signs such as those described above occurred after 20 months (SD 20.3; range 0.180.7 months) of f-TIVAP implantation and use. The average num-
Table 1 Demographic data and previous implantations of TIVAP with access site.

\begin{tabular}{|c|c|c|}
\hline Gender (\%) & female & $73(100 \%)$ \\
\hline \multirow[t]{2}{*}{ Age (years) } & median & $62.5($ SD 11.4) \\
\hline & range & $35-86$ \\
\hline Underlying malignancy (\%) & bilateral breast cancer & $73(100 \%)$ \\
\hline \multicolumn{2}{|l|}{ Mean operative time (min) } & $24.5($ SD 12.6) \\
\hline \multicolumn{2}{|l|}{ Range } & $6-75$ \\
\hline \multicolumn{2}{|c|}{ Previous catheter implantation (\%) } & $13(17.8 \%)$ \\
\hline \multicolumn{2}{|l|}{ Right cephalic vein } & $4(5.5 \%)$ \\
\hline \multicolumn{2}{|l|}{ Left cephalic vein } & $4(5.5 \%)$ \\
\hline \multicolumn{2}{|l|}{ Right subclavian vein } & $1(1.4 \%)$ \\
\hline \multicolumn{2}{|l|}{ Left subclavian vein } & $1(1.4 \%)$ \\
\hline \multicolumn{2}{|l|}{ Left femoral vein } & $1(1.4 \%)$ \\
\hline \multicolumn{2}{|c|}{ Left cephalic vein and right femoral vein* } & $1(1.4 \%)$ \\
\hline \multicolumn{2}{|c|}{ Left cephalic vein and left subclavian vein* } & $1(1.4 \%)$ \\
\hline
\end{tabular}

* Two patients who had two prior TIVAP placements had placement via the left cephalic vein and left subclavian vein or right femoral vein.

SD: standard deviation 
Table 2 Association between infection of the device and leukopenia; statistical significance for the development of DVT at the f-TIVAP access site during chemotherapy-related leukopenia.

\begin{tabular}{|c|c|c|c|c|}
\hline & Infection $(n=15 / 73)$ & p-value* & DVT $(n=14 / 73)$ & p-value* \\
\hline Leukopenia $(n=38 / 73)$ & $11 / 15$ & 0.085 & $11 / 14$ & 0.037 \\
\hline $\operatorname{DVT}(n=14 / 73)$ & $5 / 15$ & 0.146 & & \\
\hline
\end{tabular}

* Fisher's exact test

ber of punctures of every device for CTX was 10 times per patient (SD 6.0; range: 1-30 punctures). Assuming that every puncture of the port represents a relative risk of infection, we analyzed the infection rates based on frequency of port use. Patients were divided into two groups, according to the number of port punctures: patients in group 1 had fewer than eleven punctures ( $n=44 / 73$ ), patients in group 2 had more than 11 punctures $(n=29 / 73)$. The difference in infection rates between the two groups did not reach significance, but there was a strong trend toward group 2 ( $\mathrm{p}=0.084$; Fisher's exact test). Punctures for irrigations were added in a second analysis, but the difference in infection rates again did not reach significance during the period of observation ( $\mathrm{p}=0.291$; n. s.).

During CTX, 38 of 73 (52\%) women developed leukopenia. Eleven of them (29\%) were diagnosed with an infection of the device, but this only occurred simultaneous to leukopenia in three women. The association between port infection and leukopenia was not significant, but a strong trend was observed ( $\mathrm{p}=0.085$; Fisher's exact test, $\bigcirc$ Table 2 ).

\section{Thrombosis as a complication of $\mathrm{f}$-TIVAP}

$14 / 73$ (19\%) patients showed clinical signs of DVT and/or increased D-dimer levels. DVT occurred on average 21 months after f-TIVAP implantation (SD 20.9, range: 0.2-92.4 months). However, DVT was documented only in 11 women by imaging (ultrasound, venography or computed tomography/magnetic resonance tomography). Three patients were diagnosed with threelevel femoro-distal thrombosis, three patients with femoral vein thrombosis, two women with thrombosis of the inferior vena cava, two patients had localized thrombosis of the catheter tip, and one patient suffered from isolated iliac vein thrombosis. There was no statistical relationship between infection of the device and the occurrence of DVT associated with f-TIVAP (infection + DVT: $n=5 / 73 ; p=0.146$, Fisher's exact test). But 11 of 38 patients with leukopenia and receiving CTX developed DVT under leukopenia conditions at the site of the device. The incidence for leukopenia and DVT at the f-TIVAP site was significant ( $\mathrm{p}=0.037$; Fisher's exact test).

\section{Discussion}

$\nabla$

Access using the femoral vein represents an alternative approach for TIVAP implantation. Although complications such as device infection or DVT were higher than with classical implantation sites, the access path via the groin is a safer and more efficient option for patients with bilateral breast cancer.

Implantation of a TIVAP is used universally in patients requiring a permanent device for continuous administration of intravenous medications. The majority of these patients are oncology patients requiring chemotherapy or cytotoxic agents. The most common methods for TIVAP implantation are percutaneous procedure via the subclavian veins or a cut-down procedure of the cephalic veins. The cephalic vein cut-down method has the advantage that it is associated with fewer major complications than percutaneous puncture which can cause hemothorax or pneumothorax [7-9]. However, the cephalic vein cut-down approach can be difficult in patients with small cephalic veins or venous thrombosis [10]. The cephalic vein cut-down approach is a difficult technique in patients with a history of synchronous bilateral breast surgery as they may have suffered intraoperative injury to the cephalic veins or have postoperative adhesions causing venous thrombosis and making it difficult to locate the cephalic veins. The cutdown method can also increase the possibility of arm swelling in these patients. Many patients with bilateral breast cancer treated with surgery and radiotherapy are sensitive to manipulations of the chest wall, experiencing discomfort if the TIVAP system is located close to the pretreated breast.

In TIVAPs placed using a femoral approach, the catheter is inserted into the femoral vein, and the port can be implanted in the anterior thigh or abdominal wall near the anterosuperior iliac crest [11].

The port pocket site in our study was the anterior thigh in all cases. The reason for this was to minimize the amount of alloplastic material in the patient's body; the catheter length is halved compared to port placement on the anterior abdominal wall, also minimizing the clearance volume. Contrary to the recommendation of Chen and colleagues [6], the access site for the femoral vein in our collective was the right groin in 86\% (63 women). Chen's argument that a local port infection could mimic acute abdomen if the port is located on the right abdominal wall, resembling acute appendicitis, is immaterial if the port pocket is located on the anterior thigh. The reason why we preferred the right groin as an access point is the anatomic course of the iliac vein. The vein on the right side is less angulated than on the left, is not narrowed by crossing under the iliac artery, and is never affected by true May-Thurner syndrome.

The complication rates related to TIVAP implantation in the superior vena cava have been reported elsewhere in several series in the literature [8,12-13].

A retrospective study of TIVAP implantation using the standard cephalic or subclavian vein approach reported incidences of up to $1.1 \%$ for infection and $3.8 \%$ for thrombosis or catheter occlusion [8]. TIVAP implantation-related complications after using the femoral vein access route with implantation of the device relatively close to natural orifices have also been described $[6,11$, 14]. However most of these studies do not provide sufficient follow-up data.

The rate of infected devices of $21 \%$ in this series appears to be relatively high. However, the diagnosis of infection in our clinic was based on both clinical signs and proof of bacteria on microbiological testing. The diagnosis of infection in our series was therefore also based on local swelling, hyperthermia, rubor, pain around the pocket or fever rather than on microbiological findings alone. 
In the recent literature, the reported infection rates are based only on microbiological analysis. In our collective a bacterial cause was only identified in seven of 15 patients with infection (the rate of infection based on positive microbiological testing alone was $9 \%$ ). The overall rate of infection in the literature is not presented in a standardized manner. A graduation of infections could be done for subcutaneous pocket infections and bacteremia related to manipulation of the catheter $[15,16]$. The diagnosis of infection in our series was based routinely on clinical condition. When cutaneous hyperemia and swelling occurs at the catheter site (subcutaneous pocket) or the incisions, the diagnosis is clear. It is difficult to obtain diagnostic proof through microbiological investigations (cultures) in cases with suspected bacteremia because of the low sensitivity of such tests $[17,18]$, and this is the reason why the clinical condition must be used to indicate when there is a need for catheter removal. In many cases of catheter-related bacteremia, antibiotic therapy without catheter removal is an option if the patient is stable and without signs of sepsis. When there is diagnostic suspicion of infection (fever without apparent focus or catheter manipulation), peripheral blood and catheter cultures should be done. It appears logical that a port system which is used and punctured more often has a higher risk for contamination and infection. Although we could show a strong trend for this hypothesis, the value did not reach statistical significance ( $p=0.085$, n.s.).

DVT and catheter occlusion are common complications of f-TIVAP. There are a number of studies in the literature on TIVAP-associated DVT and catheter occlusion. Reported rates ranged from 5 to 50\% [19-21]. Catheter-related endothelial lesions are reported to be associated with a greater tendency to DVT in patients undergoing chemotherapy. Our collective showed a significant incidence of DVT combined with CTX-induced leukopenia $(\mathrm{p}=0.037)$, possibly because of a reduction in $\mathrm{C}$ and $\mathrm{S}$ proteins caused by the chemotherapy regimen [22]. In our study DVT occurred in $19 \%$ of patients, a rate similar to that reported in other publications [19]. No cases of pulmonary thromboembolism were observed in our study. This shows that totally implantable catheters do not have to be removed indiscriminately because of their inherent risk unless catheter obstruction is present. The clinical suspicions of DVT were based on the presence of edema, pain, and collateral circulation in the limb. Diagnostic confirmation of DVT was obtained by non-invasive imaging or/and venography. Patients were treated conservatively if the catheter still functioned. Systemic anticoagulation was done, initially using low-molecular-weight heparin. The prophylactic use of low doses of oral anticoagulant to reduce the incidence of such complications is discussed in the literature [12].

\section{Limitations of this study}

The study has several limitations; they include its retrospective design and the small number of cases reported. With regard to complications, particularly device infections, a comparison of our results with those in the literature is difficult. The recent literature only reports infection rates based on positive microbiological findings, whereas in our study an infection was already considered to be present if there were clinical signs such as local swelling, hyperthermia or rubor.

\section{Conclusion}

\section{$\nabla$}

Traditional implantation of a TIVAP through the subclavian or cephalic vein is simple and represents a common, standard procedure. However, both percutaneous puncture and the cut-down method have their limitations and risks. We describe a safe and effective method to implant a TIVAP using a femoral vein approach in selected patients. Although the complication rates, in particular rates for device infection and DVT, are relatively high, TIVAP implantation using a femoral vein approach is recommended in patients with bilateral breast cancer not suitable for cephalic vein cut-down procedures.

\section{Conflict of Interest \\ $\nabla$}

The authors declare that they have no conflicts of interest.

\section{References}

1 Kock HJ, Pietsch M, Krause $U$ et al. Implantable vascular access systems: experience in 1500 patients with totally implanted central venous port systems. World J Surg 1998; 22: 12-16

2 Teichgräber UK, Kausche S, Nagel SN et al. Outcome analysis in 3,160 implantations of radiologically guided placements of totally implantable central venous port systems. Eur Radiol 2011; 21: 1224-1232

3 Schwarz RE, Groeger JS, Coit DG. Subcutaneously implanted central venous access devices in cancer patients: a prospective analysis. Cancer 1997; 79: 1635-1640

4 Heiss P, Stroszczynski C, Gössmann H. Superior vena cava occlusion: Radiological placement of a central venous port system via femoral vein access. Radiologe 2012; 52: 455-458

5 Goltz JP, Scholl A, Ritter CO et al. Peripherally placed totally implantable venous-access port systems of the forearm: clinical experience in 763 consecutive patients. Cardiovasc Intervent Radiol 2010; 33: 11591167

6 Chen SY, Lin CH, Chang HM et al. A safe and effective method to implant a totally implantable access port in patients with synchronous bilateral mastectomies: modified femoral vein approach. J Surg Oncol 2008; 98 : 197-199

7 Seiler CM, Frohlich BE, Dorsam UJ et al. Surgical technique for totally implantable access ports (TIAP) needs improvement: a multivariate analysis of 400 patients. J Surg Oncol 2006; 93: 24-29

8 Chang HM, Hsieh CB, Hsieh HF et al. An alternative technique for totally implantable central venous access devices. A retrospective study of 1311 cases. Eur J Surg Oncol 2006; 32: 90-93

9 Di Carlo I, Cordio S, La Greca G et al. Totally implantable venous access devices implanted surgically: a retrospective study on early and late complications. Arch Surg 2001; 136: 1050-1053

10 Jablon LK, Ugolini KR, Nahmias NC. Cephalic vein cut-down verses percutaneous access: a retrospective study of complications of implantable venous access devices. Am J Surg 2006; 192: 63-67

11 Wolosker N, Yazbek G, Munia MA et al. Totally implantable femoral vein catheters in cancer patients. Eur J Surg Oncol 2004; 30: 771-775

12 Minassian VA, Sood AK, Lowe P et al. Longterm central venous access in gynecologic cancer patients. J Am Coll Surg 2000; 191: 403-409

13 Ballarini C, Intra M, Pisani Ceretti A et al. Complications of subcutaneous infusion port in the general oncology population. Oncology 1999; 56: $97-102$

14 Bertoglio S, DiSomma C, Meszaros $P$ et al. Long-term femoral vein central venous access in cancer patients. Eur J Surg Oncol 1996; 22: 162165

15 Sotir MJ, Lewis C, Bisher EW et al. Epidemiology of device-associated infections related to a long-term implantable vascular access device. Infect Control Hosp Epidemiol 1999; 20: 187-191

16 Douard MC, Ardoin C, Payri L et al. Infectious complications of long term intravenous devices: incidence, risk factors, diagnostic tools. Pathol Biol 1999; 47: 288-291

17 Blot F, Nitenberg G, Chachaty E et al. Diagnosis of catheter related bacteraemia: a prospective comparison of the time to positivity of hubblood versus peripheral-blood cultures. Lancet 1999; 354: 1071-1077 
18 Hanna HA, Raad I. Blood products: a significant risk factor for longterm catheter-related bloodstream infections in cancer patients. Infect Control Hosp Epidemiol 2001; 22: 165-166

19 Hohl Moinat C, Périard D, Grueber A et al. Predictors of venous thromboembolic events associated with central venous port insertion in cancer patients. J Oncol 2014; 2014: 743181

20 De Cicco M, Matovic M, Balestreri L et al. Central venous thrombosis: an early and frequent complication in cancer patients bearing long-term silastic catheter. A prospective study. Thromb Res 1997; 86: 101-113
21 Orci LA, Meier RP, Morel P et al. Systematic review and meta-analysis of percutaneous subclavian vein puncture versus surgical venous cutdown for the insertion of a totally implantable venous access device. Br J Surg 2014; 101: 8-16

22 Raad II, Luna M, Khalil SA et al. The relationship between the thrombotic and infectious complications of central venous catheters. JAMA 1994; 271: 1014-1016 